\title{
12
}

\section{Endemic mycosis}

Chadi A. Hage, Kenneth S. Knox, George A. Sarosi

Indiana University- School of Medicine, and Roudebush VA Medical Center,

Indianapolis, IN 46202, USA.

\section{INTRODUCTION}

Histoplasmosis, blastomycosis, and coccidioidomycosis are the three major endemic fungi in North America. Although histoplasmosis is found in all continents except Antarctica, coccidioidomycosis in South America, and blastomycosis in Africa, only in North America are these illnesses common.

These three fungal diseases share many characteristics. The causative agents are mycelial soil organisms. Illness is acquired by inhaling aerosolized spores. In the infected host, the organisms change their form, a characteristic called dimorphism. Histoplasma capsulatum and Blastomyces dermatitidis convert to a yeast form at 37 degrees $\mathrm{C}$ (thermal dimorphism), whereas Coccidioides immitis converts in tissue to a spherule that replicates by forming endospores (tissue dimorphism).

The endemic areas are large. Most of the Midwest and South Central United States is endemic for both histoplasmosis [1] and blastomycosis [2], and a large area in the Southwest United States and an adjacent area of Mexico are endemic for coccidioidomycosis [3]. All three illnesses occur in normal hosts, although histoplasmosis and coccidioidomycosis are also major opportunistic mycoses in patients with depressed cell-mediated immunity, and especially in patients with acquired immunodeficiency syndrome (AIDS) [4],[5].

Histoplasmosis, blastomycosis and coccidioidomycosis are major T-cell opportunistic infections, as demonstrated by the very aggressive course seen in patients with AIDS, in whom T-cell deficiency is most severe. 


\section{HISTOPLASMOSIS}

In the United States $H$. capsulatum var. capsulatum is responsible for the majority of the cases of histoplasmosis. H. capsulatum var. duboisii is predominantly found in south Africa and Europe (6). The spectrum of disease ranges from the asymptomatic acquisition of a positive histoplasmin skin test reaction to a rapidly fatal pulmonary or disseminated illness. It is the balance between the net immune status of the subject and the load of the infecting inoculum that determines the severity of the illness. During the past two decades, histoplasmosis has emerged as a common opportunistic infection in patients with AIDS especially those residing in endemic areas. Most of our current knowledge about this disease is derived from outbreak investigation in the Midwestern United States.

\section{The Pathogen and Epidemiology}

Histoplasma is a thermally dimorphic fungus. It has two forms; a mycelia phase and a yeast phase. Mycelia are the forms found in the environment, they are considered to be the infectious form. Mycelia display macro-and micro-conidia. Yeasts are what are found in the infected individuals. In the laboratory these two forms are inter-convertible by altering the temperatures and nutrients of the growth medium. The disease is highly endemic in the Midwestern and southeastern parts of the US. It is estimated that $50-80 \%$ of people living in the Ohio and Mississippi river valleys have evidence of remote infection with histoplasma [7]

Human and animal infections occurred after inhalation of aerosolized micro-conidia, the infecting particle, which can reach the alveolar space due to its very small size of 2 to $5 \mathrm{~mm}$. Extensive skin test surveys suggest that as many as 50 million people in the United States have been infected by $H$. capsulatum and that there are up to 500,000 new infections yearly [8].

For decades most outbreaks have occurred in urban settings. Most are associated with construction projects that disturbed contaminated soil. The most recent (and largest ever) outbreak occurred in Indianapolis, Indiana [9], associated with downtown construction of a swimming pool complex. 
Mini-outbreaks also still occur. Activities such as cutting up fallen trees or cleaning large bushes have been linked to smaller outbreaks. Histoplasmosis occurs in 2 to $5 \%$ of patients with AIDS who reside in endemic areas and up to $25 \%$ in selected areas during periods of outbreaks [10].

Pathogenesis. The lung is the portal of entry in almost every case. Due to their small size, microconidia can reach the alveolar space where they convert to the yeast form, a key step in the pathogenesis. The initial tissue response to the organism is predominantly neutrophilic, followed by an increase in alveolar macrophages [11]. After being phagocytosed, yeast survive and actually proliferate inside the macrophages, which serve then as a carrier throughout the reticuloendothelial system. Shortly after infection the yeast forms can be identified in the mediastinal lymph nodes. The fungus then gains access to the circulation. It is likely that transient self-limited fungemia occurs in most, if not all, patients. Later the yeast disseminates throughout the body to establish foci of infection in many organs, such as the liver, spleen and the adrenal glands [5].

About two weeks after infection, specific cellular immunity begins to develop. Activated lymphocytes secrete cytokines that stimulate macrophages in an attempt to boost their fungicidal activity. In mice, interleukin-12 is an important signal, leading to increased interferongamma production that confers protection against primary infection [12], [13], [14]. Tumor necrosis factor-alpha seems to be an important element in this scheme. Inhibition of TNF- $\alpha$ has been shown to alter the adaptive immune response to histoplasma infection and may predispose patients disseminated infection [12], [15], [16].

With the advent of T-lymphocyte-mediated cellular immunity, fungal replication is checked and granuloma formation begins. Healing of these lesions is accompanied by peripheral fibrosis. Central areas of encapsulated, necrotic material frequently calcify. These calcified foci manifest on chest roentgenogram as single or multiple calcified nodules. Calcified lesions are often seen in the liver and the spleen [17].

If the cell-mediated immune response is poor, the yeast continues to multiply. More macrophages are recruited, which in turn become parasitized and eventually disrupted, perpetuating the cycle [5]. A severe 
systemic illness develops, which invariably leads to death unless treated promptly and aggressively.

Clinical Manifestations. Most normal persons who are infected by the fungus remain asymptomatic. When present, symptoms vary widely from brief periods of malaise to severe, life-threatening illness. It is classically a flu-like illness with abrupt onset of fever, chills, and substernal chest discomfort. A harsh, nonproductive cough develops along with headache, arthralgias, and myalgias.

In immunocompetent individuals severe pulmonary illness develops rarely, only when the infective dose is unusually high. It may progress rapidly to the acute respiratory distress syndrome, and may lead to death from respiratory insufficiency if not treated promptly [1].

Immunocompromised individuals are more likely to progress to disseminated disease even after an infection with a smaller inoculum. The vast majority of progressive disseminated histoplasmosis is seen in patients with advanced AIDS with CD4 counts below 200 cells/mL [10].

\section{Progressive Disseminated Histoplasmosis}

Most patients with progressive disseminated Histoplasmosis present with fever, chills, weight loss, cough and progressive dyspnea [18]. On physical examination, patients are febrile and acutely ill. Hepatosplenomegaly may be present. Laboratory evaluation shows anemia, leukopenia, and thrombocytopenia. In extremely ill patients the syndrome of disseminated intravascular coagulation may be seen. Up to $20 \%$ of patients present with severe septic shock, respiratory failure and progress to multi-organ failure. This syndrome represents an advanced stage of the illness and is usually seen when diagnosis and appropriate therapy were delayed. Mortality is very high with this scenario.

The chest roentgenographic findings are variable, ranging from normal to diffusely abnormal, with reticulonodular pattern being the most frequently reported finding. Pleural effusions are rarely seen [19]. The radiographic findings are very similar to those seen with Pneumocystis carinii pneumonia [4] ( Figure 1). 


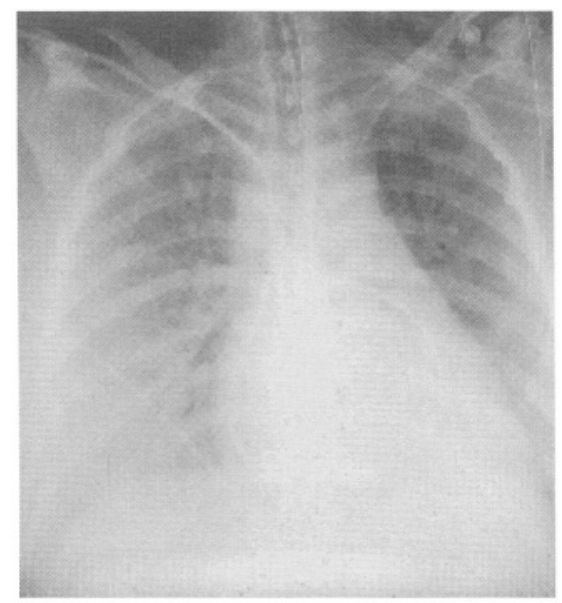

Figure 1

Chest X-ray of a HIV + patient with progress disseminated Histoplasmosis.

Peripheral blood smear may show phagocytoses yeast in some of patients with severe disease. Biopsy material from the bone marrow and other involved tissue shows collections of macrophages full of intracellular yeast or, in the most severe instances, widespread necrosis with large numbers of organisms lying loose in the extracellular debris. There is little, if any, evidence of granuloma formation [20] . Virtually all patients with this form of the illness have some degree of T-cell defect. Before the modern era of widespread use of cytotoxic agents and glucocorticoids, many patients had underlying Hodgkin's disease, a well-known example of naturally occurring T-cell immune deficiency [5], [4].

The most severe form of progressive disseminated histoplasmosis (PDH) occurs in patients with AIDS with profound T-cell dysfunction [21]. In fact, most cases of PDH now occur in AIDS patients, and most occur in highly endemic areas [22], [23]. Newer biological therapies for Rheumatoid Arthritis and other auto-immune disorders have added a new pool of immunosuppressed patients at risk for tuberculosis and also histoplasmosis and other T-cell opportunistic fungal infections.

In most instances, exposure of an immunosuppressed person to an infected aerosol is the antecedent event preceding PDH. In the recent large outbreak in Indianapolis, most patients who were immunocompromised when they 
developed primary histoplasmosis progressed to PDH [23]. In particular, patients with AIDS nearly always progressed to PDH [10].

In other cases, the onset of PDH is temporally related to intense immunosuppression, most commonly progression of AIDS or therapy with high doses of glucocorticoids. In some of these cases, reactivation of dormant histoplasmosis may be the mechanism of infection [4]. Patients with AIDS who develop PDH after long residence in New York City or San Francisco are clear examples of such endogenous reactivation because primary infections are never seen in these cities.

Some patients with PDH present with a subacute to chronic illness. They may have a chronic wasting disease with anorexia, weight loss, and lowgrade fever. Mucosal and mucocutaneous junction ulcers may occur in the mouth, oral pharynx, rectum, and glans penis. Adrenal involvement may cause Addison's disease [24]. Biopsy material from involved tissues shows well-formed epithelioid granulomas, and only a diligent search will reveal rare organisms. Demonstration of organisms almost always requires special stains [5], [20]. The disease may be systemic or involve only one organ. This more chronic form of PDH generally occurs in patients who are less immunosuppressed than the patients who develop more fulminant PDH.

Central nervous system histoplasmosis is rare and may present as chronic meningitis or intracranial histoplasmoma [25]. Endocarditis can also occur, involving either the aortic or mitral valves. Vegetations are usually large, and emboli are common. Endocarditis may occur on prosthetic or previously normal valves. Recently, histoplasma involvement of abdominal aortic aneurysms has been reported in a few patients with the chronic form of PDH [26].

\section{Diagnosis}

The gold standard of diagnosis is culture of the fungus from biologic material. Cultures are time consuming and one cannot wait for them in the management of cases with severe PDH. Delay in diagnosis while awaiting results of fungal cultures may lead to a fatal outcome in more severe cases Isolation of $H$. capsulatum may occur within 1 week in a minority of patients but usually takes several weeks. 
Serologic studies of Histoplasmosis are seldom useful in the management of PDH. Positive serology does not predict development of PDH in patients undergoing bone marrow transplantation [27]. The complement fixation test is negative in up to $50 \%$ of patients with PDH. Immunodiffusion fails to identify up to $50 \%$ of patients with acute histoplasmosis and usually does not reach maximum positivity for 4 to 6 weeks after exposure [28]. Their main drawbacks are imperfect sensitivity and lack of timeliness. Several weeks must pass before they become positive. By that time, most patients have either recovered or have required other more invasive methods of diagnosis because of rapidly worsening disease

There are two ways to make a rapid diagnosis of PDH, sampling and examination of likely infected tissue with the use of special stains and the use of the ultrasensitive assay for fungal antigens.

Mycologic Studies. Bronchoscopy is an important diagnostic tool, especially for PDH. Specimens obtained from bronchoscopy have a high but poorly defined yield in severe primary histoplasmosis with progressive ARDS and especially in PDH in AIDS, when diffuse infiltrates are one of the clinical features. In highly selected series the diagnostic yield of bronchoscopy for diagnosis of histoplasmosis in an endemic area is about $60 \%$ in patients with infiltrates [29]. In a strictly AIDS population in Indianapolis, Indiana, fungal stains performed on bronchoalveolar lavage fluid provided a rapid diagnosis in $70 \%$ of patients; diagnostic yield increased to $89 \%$ when culture results were included. In that series, $22 \%$ of patients had co-infections or alternative diagnoses that were detected by BAL and would not have been detected if Histoplasma antigen testing had been the sole diagnostic test [30]. Use of cytologic examination without special fungal staining (silver, PAS) may explain the lower yield of BAL reported in series from non-endemic areas [31]. It is likely best to do a battery of stains including a silver stain. Although transbronchial biopsy is not mandatory at the time of bronchoscopy and BAL, histopathology does appear to enhance the diagnostic yield [31]. The fungus is difficult to see on standard hematoxylin and eosin stains; special stains (usually a silver stain) are needed. Special stains are particularly important when well-formed granulomas are present because of the paucity of organisms in such cases.

In patients with suspected PDH, sampling of the reticuloendothelial system is often effective for diagnosis. Bone marrow biopsy is likely the best and 
safest method [20] . In heavily parasitized samples, a direct smear of the bone marrow, stained with a supravital stain such as the Giemsa stain, usually gives a rapid diagnosis (Figure 2). On permanent histologic sections, the fungus is difficult to see on standard slides prepared with hematoxylin and eosin stain. It is best to go directly to special stains, usually one of several modifications of the silver stain or the PAS stain.

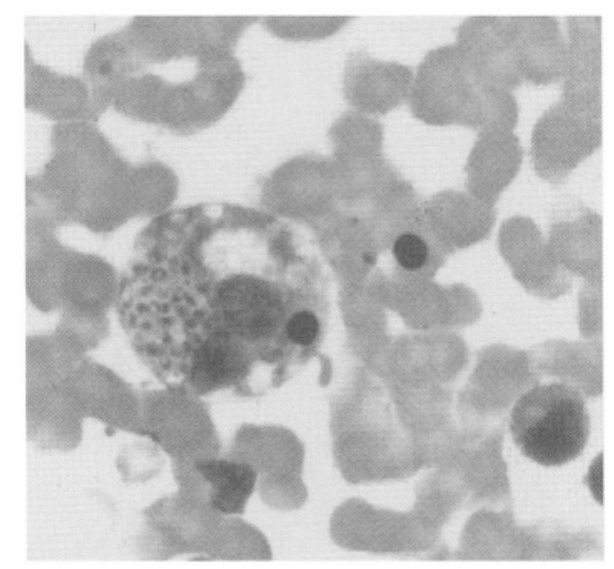

Figure 2

Intraphagocytic yeasts seen on a bone marrow aspirate.

Recently, the role of blood cultures in diagnosis of PDH has expanded. The lysis-centrifugation system increases sensitivity. In AIDS patients with $\mathrm{PDH}$, the density of organisms is higher than in other immunosuppressed patients, and blood cultures are particularly useful, yielding a diagnosis in up to $90 \%$ of cases. In fact, in AIDS patients with PDH typical intracellular organisms can be seen directly on peripheral blood smears (buffy coat preparations) up to 50\% of the time. Bronchoalveolar lavage also has a very high yield, both by direct smear and by culture in AIDS patients with high burden of organisms. Bronchoalveolar lavage offers the additional advantage of ready diagnosis of other opportunistic infections that are usually in the differential diagnosis, including $P$. carinii pneumonia.

Antigen Detection. Another approach to diagnosis of fungal infections is the use of the ultrasensitive assays for fungal antigens. This test is very useful when the burden of the infection is high. Detection of the 
histoplasma antigen in body fluids permits rapid diagnosis of PDH. The specificity of antigen detection is greater than $98 \%$. There is, however, known cross reactivity with the other endemic fungi such as African histoplasmosis, blastomycosis, paracoccidioidomycosis, and Penicillium marneffii infection [32]. Sensitivity is lower in patients who are moderately immunosuppressed. However, the high density of organisms in AIDS patients with PDH makes antigen testing extremely useful in that setting.

Antigen testing is more sensitive on urine than serum. It is positive in either urine or serum in up to $95 \%$ of patients with PDH complicating AIDS [30]. Levels of histoplasma polysaccharide antigen in urine and serum also are useful for following the course of treatment and for predicting relapses [33], [34]. The test is done reliably in a single reference laboratory in Indianapolis, which makes it a "send out" for many institutions.

\section{Treatment}

Severe cases of PDH in non-AIDS patients are best treated promptly and aggressively with amphotericin B to a total dose of $40 \mathrm{mg} / \mathrm{kg}$. Itraconazole (400 mg/day in a single daily dose) can be used successfully for patients with mild to moderate disease [35]. Sequential therapy for severely ill patients with amphotericin B to clinical improvement followed by 6 months of itraconazole is also being used but is not well studied.

AIDS patients are treated differently from other PDH cases. Relapse is expected if treatment is stopped. All patients require induction therapy to control symptoms and then maintenance therapy. Amphotericin B is used initially for all moderately and severely ill patients. After clinical response, treatment is changed to itraconazole ( $400 \mathrm{mg}$ daily for 6 or more weeks, then $200 \mathrm{mg}$ daily until sustained recovery of the immune system) [36], [37]. There is emerging data that support the discontinuation of maintenance Itraconazole in patients with HIV who recover their immune system [38].

Itraconazole can be used from onset for mild cases[39]. Other maintenance strategies for those intolerant of itraconazole include weekly amphotericin $\mathrm{B}$ infusions or fluconazole at doses of at least $400 \mathrm{mg} / \mathrm{day}$. Ketoconazole therapy is ineffective for maintenance therapy, and fluconazole even at high daily doses (400 to $800 \mathrm{mg}$ ) is less effective than itraconazole [40]. In a 
recent double blind, randomized trial liposomal amphotericin B (L-AMB) was somewhat more effective than AMB in both time of response and survival, although the differences were not statistically significant [41]. In this study both types of AMB were used for fourteen days before switching to oral itraconazole therapy.

\section{BLASTOMYCOSIS}

Blastomycosis is an illness caused by the thermal dimorphic fungus Blastomyces dermatitidis. The spectrum of disease ranges from the asymptomatic acquisition of the fungus to a rapidly progressive and lifethreatening respiratory or disseminated illness.

\section{Epidemiology}

Blastomycosis is most common in the central and south-central United States [42]. The proposed endemic area includes much of the central, south central, and southeastern United States, beginning near the MinnesotaNorth Dakota border and extending eastward and southward. The southeastern limit extends to South Carolina but not to Florida. This area overlaps most of the endemic area of histoplasmosis [1]. The northern limit, however, extends further. Northern Minnesota and northern Wisconsin and also the adjacent Canadian provinces of Ontario and Manitoba are endemic for blastomycosis but are free of histoplasmosis [43], [44] .

Similar to $H$. capsulatum, $B$. dermatitidis is a soil-dwelling fungus. Infection occurs by inhalation of airborne spores. Infected individuals develop a positive blastomycin skin test reaction or the in vitro correlate of delayed hypersensitivity. As with histoplasmosis, isolated microfoci of high infectivity exist in a large endemic area. Outbreaks often occur during activities such as hunting, camping, or canoeing in wooded or swampy environments [45]; these are most common when soil temperatures have been increasing for several days and when there is rain on the day of exposure. For sporadic cases, residence close to water in a highly endemic area and recent excavation activity are risk factors [46]. Dogs are also susceptible to blastomycosis. Canine blastomycosis is a well-recognized entity in veterinary practice in the endemic areas. The recognition of canine 
cases in a community should alert physicians that human blastomycosis may be present in their geographic area [47].

\section{Pathogenesis}

At ambient temperatures, the fungus grows as an aerial mycelium. When foci of actively growing blastomyces are disturbed, small 2- to 5- $\mu \mathrm{m}$ spores become airborne and an infective aerosol is formed. These infecting particles then may be inhaled by humans or by other mammals disturbing the site. Some spores may escape the nonspecific defenses of the lung and reach the alveoli.

The initial inflammatory response is neutrophilic. As the organism converts to the parasitic yeast form and begins to multiply, large numbers of yeast are seen, surrounded by neutrophils. Following this macrophages increase in number. Eventually, as specific cellular immunity develops, there are giant cells and well-formed epithelioid granulomas. In contrast to histoplasmosis, the neutrophilic component of the inflammatory response does not disappear completely, and the histopathologic examination often shows a mixed pyogenic and granulomatous response, even in chronic cases [2].

It would be misleading, however, to think that there is a "characteristic" tissue response in blastomycosis. Occasionally, the neutrophilic component is minimal and the granulomas are noncaseating, producing a picture similar to that of sarcoidosis. In contrast, granulomas are sometimes entirely absent in overwhelming infections. The entire inflammatory reaction consists of neutrophils, and the histopathologic picture mimics that of bacterial infection.

The histopathologic response in cutaneous blastomycosis is striking. The stratified squamous epithelium becomes markedly hyperplastic, with exaggerated downgrowth of the rate pegs. Within these fingerlike projections are a number of microabscesses. The same hypertrophic tissue response is seen when the disease involves the oropharynx or the larynx. The histopathologic appearance may superficially resemble carcinoma. The characteristic organisms, seen best with special stains, provide a diagnosis. 
The initial inflammatory and immune response may confine the infection to the lungs and the hilar lymph nodes. It is likely (but not proven) that selflimited early fungemia does not occur as often as it does in histoplasmosis. In some instances, however, the organism spreads beyond lung and the hilar nodes. Dissemination is usually to skin, bones, prostate, and meninges but can be seen in any organ [2], [48] .

The incubation time for blastomycosis is longer than for histoplasmosis and more variable. In the Eagle River outbreak, in which time of exposure was short and precisely defined, the median incubation period was 45 days, with a range of 21 to 106 days [49], [50].

\section{Clinical Manifestations}

The portal of entry is almost always the lung, and the primary illness is a lower respiratory infection. Some patients have an acute illness that resembles bacterial pneumonia, in contrast to acute pulmonary histoplasmosis, which more closely mimics influenza. The onset of symptoms is abrupt, with high fever and chills, followed by cough that rapidly becomes productive of large amounts of mucopurulent sputum. Pleuritic chest pain may occur.

This acute onset is common in an outbreak setting, but also may be seen in sporadic cases. In most sporadic cases, however, the onset of clinical symptoms is more gradual. The patient presents with a low-grade fever, productive cough, and weight loss [48] [51]. Lung cancer or tuberculosis are highest in the differential diagnosis, rather than bacterial pneumonia, depending on the roentgenographic findings.

Physical examination is usually unremarkable except for fever. Auscultation of the chest in patients who have segmental or lobar infiltrates may show crackles and focal consolidation; more often the physical examination is negative. Skin lesions are highly variable in appearance, ranging from subcutaneous nodules and abscesses to papules to ulcers with heaped up borders mimicking squamous cell cancers. Perhaps the most characteristic lesion has irregular borders and a crusted surface, varying in size from 1 to 10 or more centimeters. Skin lesions may be single or multiple and may occur in crops of several new lesions daily or every few days if the disease is rapidly disseminating. 
Routine laboratory tests are seldom helpful. In cases resembling acute bacterial pneumonia, the white blood cell count is elevated, and frequently there is a shift to the left toward earlier forms in the granulocyte series.

There is no "characteristic" chest roentgenographic pattern in blastomycosis [52]. Lesions may vary from single or multiple round densities throughout both lung fields to segmental or lobar consolidation. Severe pulmonary infections can present with diffuse infiltrates, nodular, interstitial, or even alveolar (Figure 3). The diffuse alveolar infiltrates are identical to acute lung injury (as in acute respiratory distress syndrome) of diverse cause. Mass-like perihilar infiltrates, especially on the right side, are common and are often misinterpreted as neoplastic. On the lateral chest roentgenogram, the mass-like infiltrate is usually behind the hilum, in the apical-posterior segment of the lower lobe. Hilar lymph node involvement may occur but is not nearly as common as in histoplasmosis. Cavities may occur during the acute phase of the illness and usually close during successful treatment. Unlike histoplasmosis, calcification due to healed blastomycosis is rare.
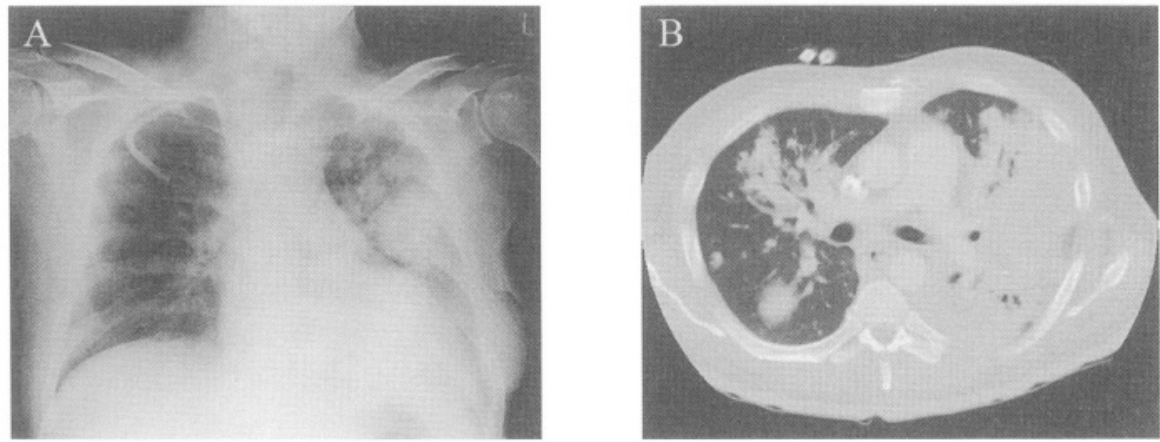

Figure 3

47 year old man who failed antibiotic therapy for a community acquired pneumonia, presenting with a rapidly progressive respiratory failure. Bronchoalveolar lavage recovered Blastomyces dermatitidis. 
Extrapulmonary spread of the fungus may occur during the acute, symptomatic phase of the illness. In some instances, only the distant lesion (usually skin or bone) is symptomatic.

The skin and the bony skeleton are the most common sites of symptomatic extrapulmonary spread. The prostate gland, meninges, oral pharynx, larynx, and abdominal viscera, including the liver and the adrenal glands, are involved less frequently [2].

Blastomycosis can present as a progressive infection in patients with T-cell defects, including organ transplant recipients and other patients being treated with high-dose glucocorticoids and other immunosuppressive therapy for malignant and nonmalignant disorders. As with histoplasmosis, the disease can often be cured with amphotericin B in patients with intermediate degrees of immunosuppression.

Blastomycosis is much less common in AIDS and other T-cell-deficient conditions than are histoplasmosis and coccidioidomycosis. This is probably because exposure to this fungus, while immunosuppressed, is less common and because there is a smaller reservoir of patients with remote healed infection waiting to relapse should T-cell function markedly decline [53].

Blastomycosis can also occur in AIDS, usually with a CD4 count below $200 / \mu 1$. Infection is particularly severe, and cure is not likely. Maintenance therapy is required for those who respond to initial treatment. Patients with AIDS are likely to progress with widespread dissemination of the infection with multi-organ involvement. Patients may present with sepsis like picture. Meningitis or brain abscess are common in this setting and it is associated with a high early mortality [54].

\section{Diagnosis}

The easiest and most rapid method of diagnosis is examination of expectorated sputum or aspirated pus after $10 \%$ potassium hydroxide digestion [2]. The characteristic large (8- to $20-\mu \mathrm{m})$ organism is easily identified. The yeast is single budding, with a broad neck of attachment between the parent and the daughter cells. The wall is thick and is double refractile, and there are multiple nuclei. Other direct fungal stains including 
periodic acid Schiff (PAS), calcoflour white, and silver stains are more sensitive. Another sensitive technique for rapid diagnosis of blastomycosis on direct sputum smears is cytologic analysis with the standard Papanicolaou stain (Figure 4). The direct techniques are probably complementary and examining multiple sputum samples increases diagnostic yield [55].

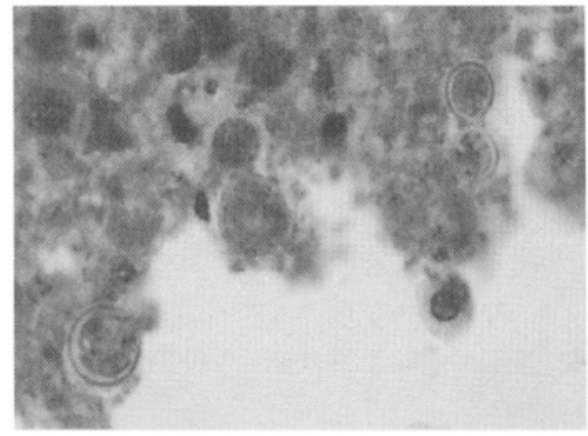

Figure 4

Papanicolaou stain of a sputum specimen from a patient with rapidly progressive respiratory failure after a community acquired pneumonia, showing the large budding yeast with broad neck of blastomyses.

Bronchoscopy is useful when patients are unable to expectorate adequate sputum, and when urgent diagnosis is needed because of rapid pace of the illness. In one study, bronchoscopy (specimens obtained included bronchoalveolar lavage (BAL) in 64\% and bronchial washings in all) was diagnostic in $92 \%$ of patients when culture results were included in the final analysis [56].

For patients who are acutely ill or have an ARDS-like picture, rapid diagnosis is crucial and can only be achieved by direct examination of respiratory secretions. If direct sputum smears are negative or not possible, then bronchoscopy should be done urgently with BAL and bronchial washings sent for both direct fungal stains (some combination of $\mathrm{KOH}$, calcofluor white, silver stain, PAS and cytology preparation) and for culture [57]. The cytology laboratory should be informed whenever there is high clinical suspicion of infection. Cellblocks of concentrated BAL fluid can be done to maximize the yield of the submitted specimens.

Histopathologic examination of biopsy material is also an excellent way to establish the diagnosis. The decision whether or not to perform 
transbronchial biopsies at the time of initial bronchoscopy will likely depend on contraindications in any given patient that give added risk beyond risk of BAL. This is particularly true in critically ill patients. If blastomycosis is being considered in this setting, bronchoscopy with BAL can be the initial procedure, reserving transbronchial biopsy for cases with no diagnosis from a safer and easier first procedure. Standard hematoxylin and eosin stains do not stain the fungus and special stains are required. The periodic acid-Schiff stain preserves morphological detail, but silver stains are more commonly used and likely have better sensitivity.

Identification of the fungus by culture is not difficult, but it is slower. Growth may occur as early as 5 to 7 days but often takes several weeks. Exoantigen testing can provide positive identification as soon as good growth is established. Formerly, positive identification required conversion of the mycelial culture to the yeast phase of growth, adding 1 or more weeks of delay.

Serologic testing is of limited value in diagnosis of blastomycosis. They are positive in about a quarter of cases. Most cases of blastomycosis are diagnosed by smear, culture, and histopathology rather than by serological tests.

\section{Treatment}

Severely ill patients with pulmonary blastomycosis require immediate and aggressive treatment with amphotericin B.

Itraconazole is highly effective for blastomycosis and is the treatment of choice for most patients with pulmonary and nonmeningeal disseminated disease; oral therapy with $400 \mathrm{mg} /$ day for 6 months successfully treats most patients with mild to moderate pulmonary disease, skin disease, and bone disease.

Amphotericin B (dosing as described for histoplasmosis; total cumulative dose $2000 \mathrm{mg}$ ) is preferred for a small minority of severely ill patients, including all patients with diffuse infiltrates and severe gas exchange abnormalities. Patients with edematous lobar pneumonia (bulging fissures), extremely toxic patients, and patients who are rapidly disseminating should all receive AMB. For these severe infections, sequential therapy with AMB 
to clinical improvement (usually 500 to $1000 \mathrm{mg}$ total dose) followed by 6 months of oral itraconazole is often used and is effective though not well studied. This approach is also used for AIDS patients. Patients with AIDS and blastomycosis are not permanently cured. Life-long maintenance therapy is needed after induction therapy to improve symptoms.

Meningeal blastomycosis is always treated with systemic amphotericin B therapy in standard doses. L- AMB achieves higher brain tissue levels in animals and may be preferred. Fluconazole is overall less potent for blastomycocis than itraconazole but penetrates CNS much better. High dose fluconazole (often in combination with L-AMB) has been used for central nervous system blastomycosis. Voriconazole is theoretically attractive but has not been studied. Intracisternal amphotericin B has been used anecdotally in addition to systemic therapy for selected patients, but it is uncertain whether there is additional benefit [58], [59] . Intracisternal therapy is used less often now that there is a wider range of therapy.

\section{COCCIDIOIDOMYCOSIS}

Coccidioidomycosis is the illness caused by the tissue-dimorphic fungus Coccidioides immitis. Although most infections are mild and self-limited, the spectrum of illness includes life-threatening pulmonary disease and widely disseminated systemic disease with a high mortality rate. Differences between it and histoplasmosis and blastomycosis include different endemic areas, higher frequency of meningeal infections, and poorer response to all antifungal therapy, including amphotericin B.

\section{Epidemiology}

The endemic area for Coccidioidomycosis in North America is the southwestern United States and the contiguous areas of northern Mexico. The endemic area of the United States includes central and southern California and extends eastward to Arizona, New Mexico, and western Texas.

In nature, the fungus grows as an aerial mycelium with septate hyphae. Alternating cells form thick-walled barrel-shaped structures called arthroconidia, with empty cells in between. When a natural site is 
disturbed, the mature arthrospores easily detach and become airborne, producing an infective aerosol.

The risk of infection is greatest during the hot dry summers. Strong winds can carry the arthrospores for long distances. A huge wind storm that blew north from the San Joaquin Valley in 1977 caused a major outbreak of coccidioidomycosis in Sacramento, far north of the usual endemic area [60]. Not surprisingly, occupations and activities with exposure to the soil carry the greatest risk for infection, including construction work, farm labor, and working on archeological digs [61].

\section{Pathogenesis}

After inhalation, some arthrospores evade the nonspecific lung defenses and reach the alveoli, where germination begins. The arthrospores develop into spherules, the tissue phase of the fungus. Spherules are large, round, thick-walled structures that vary in diameter between 10 and $80 \mu \mathrm{m}$. Reproduction of the fungus occurs within the spherule. The cytoplasm of the spherule undergoes progressive cleavage, forming numerous endospores within the spherule. Once a spherule matures, it bursts and releases the endospores into the surrounding tissues. Each endospore can become a new spherule and thus repeat the process.

The initial inflammatory response to inhaled arthrospores is neutrophilic. Resident alveolar macrophages also phagocytose the arthrospores and prime specific $\mathrm{T}$ lymphocytes, which multiply, recruit more macrophages, and arm the macrophages, engaging specific cell-mediated immunity. Even though many well-formed granulomas are seen, the neutrophilic inflammatory exudate does not disappear. Histopathologically, there is a mixed granulomatous and suppurative reaction more similar to blastomycosis than to histoplasmosis [3]. Granuloma formation is important for successful limitation of the infection. Good outcome correlates with preponderance of well-formed granulomas.

Most primary infections are asymptomatic or relatively mild. The fungus usually remains localized to the lung and hilar lymph nodes. Dissemination occurs in less than $1 \%$ of patients. Hematogenous spread can affect many tissues, including the skin, bones, lymph nodes, visceral organs, and 
meninges [62]. Meningitis is the most feared clinical syndrome with an ominous prognosis [63].

\section{Clinical Manifestations}

Except for rare instances of inoculation coccidioidomycosis, the portal of entry is the lung. About $60 \%$ of individuals with primary pulmonary infection remain totally asymptomatic. In the remaining $40 \%$, the spectrum of disease ranges from a mild, influenza-like respiratory illness to a severe, life-threatening pneumonia [62].

The clinical symptoms and their severity are variable. Common symptoms include cough, fever, and pleuritic chest pain. Cough may be nonproductive, or there may be small amounts of mucopurulent sputum. True rigors are not common. Headache, common during the acute phase of the illness, is nonspecific. Severe headache is always worrisome, however, because coccidioidal meningitis often becomes clinically apparent during the early part of the illness. If meningitis is suspected, a lumbar puncture should be performed immediately.

Several dermatologic aspects of acute coccidioidomycosis are important. A mild nonspecific so-called toxic rash occurs in many patients [61], [64]. It is an erythematous macular rash that occurs early during the illness, before the skin test turns positive. Erythema nodosum and erythema multiforme are other skin manifestations of primary coccidioidal infection. Together with fever and arthralgias, these skin lesions are part of a variable symptom complex first recognized by locals in the San Joaquin valley of south central California and labeled "valley fever".

More than $75 \%$ of patients with primary coccidioidomycosis have an abnormal chest roentgenogram. The most common roentgenographic abnormality is a single or multiple areas of patchy pneumonitis. The ipsilateral hilar nodes are enlarged in about $25 \%$ of patients [65] . Hilar adenopathy may also be seen without recognizable parenchymal disease (Figure 5). 


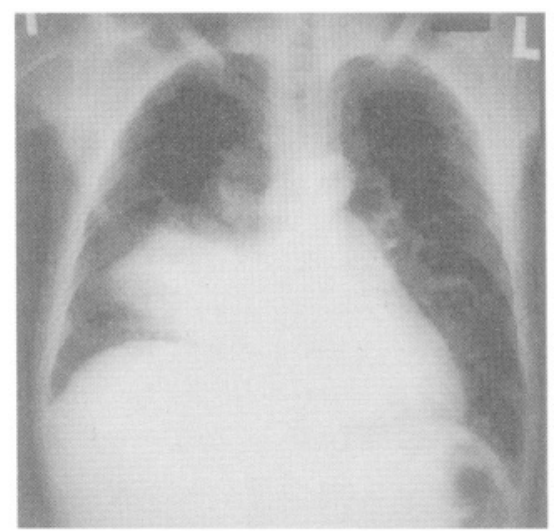

Figure 5

Acute pulmonary Coccidioidomycosis presenting as an acute community acquired pneumonia.

This primary complex usually heals rapidly. Necrosis in the center of a pneumonic lesion may produce cavitation [66]. This is often accompanied by minor hemoptysis, which may be alarming but is seldom lifethreatening. Hemoptysis as a late complication is uncommon but can be life-threatening, in contrast to the minimal bleeding that is often seen as the cavity forms. Another complication is rupture of the cavity with development of a pyopneumothorax.

In rare instances, primary pulmonary Coccidioidomycosis is not self-limited but progresses within the lung. Symptoms are fever, cough, and weight loss. The chest roentgenogram shows progression of the infiltrate and variable involvement of the hilar nodes[65]. This form of pulmonary Coccidioidomycosis is dangerous and augers impending dissemination. Most of the patients with progressive pulmonary disease are either immunosuppressed or belong to groups at high risk for dissemination.

The primary pulmonary infection usually either resolves completely or stabilizes. Rarely does a patient die when the disease is restricted to the lung. In some individuals, however, the fungus spreads widely throughout the body, resulting in a systemic infection known as disseminated Coccidioidomycosis.

Patients receiving glucocorticoid or cytotoxic or newer immune modulating therapy for malignant or nonmalignant diseases are at risk of dissemination. This is especially true for recipients of renal and other organ 
transplant and patients with AIDS [67], [68]. The excess risk of coccidiodomycosis in organ transplant recipients in highly endemic areas has led to targeted prophylaxis to prevent re-activation whenever there is a history of coccidiodal infection or positive serologic results on pretransplant screening [69]. There are other well-recognized risk factors for dissemination. Race and ethnicity are important. Disseminated coccidioidomycosis is more likely in blacks, Filipinos, and native Americans than in whites. Male gender is also a risk factor, as is diabetes mellitus. The very young and the very old are more likely to have dissemination[62]. There is much anecdotal information suggesting that coccidioidomycosis during the third trimester of pregnancy may be a severe illness with rapid dissemination.

Dissemination from the primary pulmonary focus tends to occur early, usually within a few months after a symptomatic pulmonary infection. In some patients, however, the findings of disseminated disease are the first manifestations of coccidioidomycosis, presumably because the preceding pulmonary infection was sub-clinical.

Dissemination may involve any organ in the body. The skin is one of the most common sites of dissemination and is involved in most patients some time in the course of the disease. Involvement of the bones is the next most common manifestation of disseminated coccidioidomycosis. Osteomyelitis may be either the sole evidence of extrapulmonary spread or part of a more widespread dissemination. Bone disease is usually restricted to one or two sites, but occasionally as many as eight separate lesions may be present.

Meningitis is the most dreaded complication of coccidioidal dissemination. Between one third and one half of all patients with disseminated disease have meningitis, frequently as the only obvious extrapulmonary site. The onset of meningitis may be subtle, with only mild headache and minimal alteration of mental functions. Striking boardlike nuchal rigidity, as in purulent meningitis, is seldom seen [63]. In fact, the findings of meningitis can be so minimal that all patients with dissemination at other sites should have a diagnostic lumbar puncture to exclude meningitis. Involvement of the base of the brain is characteristic. As the disease progresses, an exudate frequently obstructs the aqueduct of Sylvius and the foramina of the fourth ventricle, producing hydrocephalus. When obstruction occurs, the patient's clinical condition suddenly worsens, with diminished level of consciousness and the development of papilledema. The cerebrospinal fluid shows 
characteristics of chronic meningitis: predominantly mononuclear cell pleocytosis, increased protein, and decreased glucose. Occasionally, eosinophils are present in the cerebrospinal fluid. If present, they are a valuable clue to the possible coccidioidal nature of the chronic meningitis.

When coccidioidomycosis complicates HIV infection, the severity depends on the residual immune competence of the host. With near-normal CD4 lymphocyte counts, coccidioidomycosis is not significantly different from the disease seen in normal hosts. When the CD4 count falls below 250 cells $/ \mathrm{mL}$, disseminated disease tends to be severe and rapidly progressive. Patients usually have high fever, complain of dyspnea, and are hypoxemic; chest roentgenograms often show diffuse reticulonodular infiltrates with nodules $5 \mathrm{~mm}$ or greater in diameter (Figure 6). Diffuse macronodular pulmonary infiltrates are present in less than one per cent of non-AIDS patients with disseminated coccidioidomycosis, but in up to $50 \%$ of advanced AIDS patients with this condition. Meningeal disease is present in up to $25 \%$ of the patients [70], [71] .

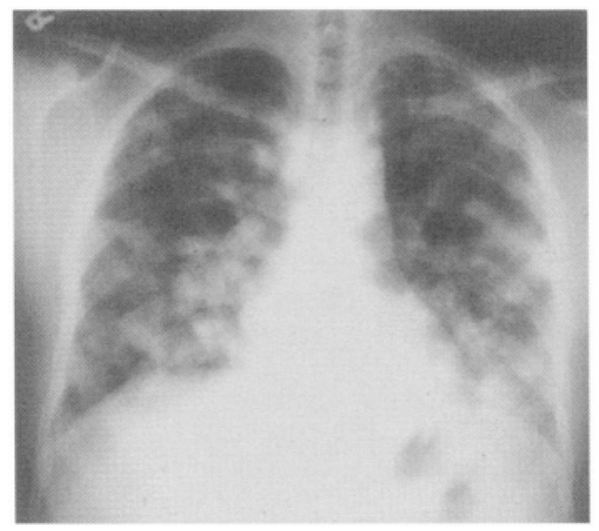

Figure 6

Disseminated Coccidioidomycosis in an HTV infected patient.

\section{Diagnosis}

Mycologic Studies. Direct examination of sputum and other respiratory specimens (or pus from a non-pulmonary site) may reveal the diagnostic 
spherules. Direct smears have highest utility in patients who produce copious sputum or have multi-lobar infiltrates [72] . Bronchoscopy is often performed in selected cases. In one study bronchoscopy was diagnostic in $69 \%$ of patients (compared to $32 \%$ for sputum stains and cultures) when patients with solitary pulmonary nodules on chest radiograph were excluded from analysis [73]. This study also showed usefulness of a postbronchoscopy sputum and equivalent sensitivity for Papanicolaou and silver staining. The airway can be examined at the time of bronchoscopy and may be abnormal, providing clues to the diagnosis [74].

Bronchoscopy is typically performed in patients who are immunosuppressed and severely ill, especially if they have diffuse infiltrates on chest radiograph. Multiple infections often co- exist, adding additional value to diagnostic bronchoscopy early in the course of illness [75], [76] . Bronchial washings and bronchoalveolar lavage fluid should be sent for cytology, fungal stains, and culture. In a recent study of an AIDS patient in Phoenix, Arizona, the Papanicolaou stain was the most useful direct test (when compared to $\mathrm{KOH}$ and calcofluor white) for rapid diagnosis of pulmonary coccidioidomycosis and was even positive in two patients with negative cultures [77]. Histopathologic examination of biopsy material is extremely helpful. When mature spherules (visible on standard hematoxylin and eosin stained tissue sections) are seen, the diagnosis is secure. More commonly, only endospores, immature spherules or spherule fragments are present. Therefore fungal stains such as a silver stain should always be used in addition to hematoxylin and eosin staining. In one study, transbronchial biopsy yielded a specific tissue diagnosis of coccidioidomycosis in eight of eight patients.

Cultural identification of the fungus is not difficult but is hazardous to laboratory personnel. Isolation should be attempted only under rigid biohazard protection. Traditional laboratory methods for identifying culture isolates require conversion of mycelial-phase cultures to the tissue phase either by animal inoculation or directly by the use of slide cultures. Now immunodiffusion tests are performed directly on the supernatants of liquid mycelial-phase cultures. This method of identification (called exoantigen testing) is safer, simpler, and faster [78]. Positive identification of a coccidioidal isolate can sometimes be made by day 5 , although it usually takes longer. 
Serologic Studies. Because cultural identification is slow and even somewhat dangerous, serologic tests have been developed that facilitate rapid diagnosis [3] [79]. A tube precipitin tests for detection of Ig M antibodies is positive in $90 \%$ of patients by the third week (negative only in very mild infections). Because the test usually reverts to negative within 3 months, it is quite specific for recent infection[3]. Currently, an immunodiffusion test for IgM has largely replaced the tube precipitin test. The immunodiffusion test measures the same antibodies, but it is easier to perform.

The most important serodiagnostic test is the complement fixation (CF) test. CF antibodies are of the $\operatorname{IgG}$ class and appear later than IgM antibodies. In most symptomatic patients, the CF test is positive by 2 months and remains positive for several months or longer [79]. The test is highly specific but is not sensitive. Most asymptomatic skin test converters never have CF titers over 1:8, which is the threshold for a positive result. Most symptomatic patients have titers of 1:8 or 1:16. Titers of 1:32 or higher are generally associated with more severe infections and poorer prognosis. In the classic studies of Smith and colleagues [79], many patients with these high titers either had already undergone or were about to undergo dissemination. However, other patients with disseminated coccidioidomycosis did not have high titers. Also the cutoff of a 1:32 CF titer as a harbinger of dissemination never transferred perfectly to other laboratories that did not use the same method or the same antigen. A single CF titer, no matter how high, should never be used to make a diagnosis of disseminated coccidioidomycosis. Nonetheless, a steadily rising titer should raise the suspicion of disseminated coccidioidomycosis and prompt further tests (including bone scan, spinal tap, or both when appropriate) to better define the extent of disease.

\section{Treatment}

Because dissemination is more likely in immunosuppressed patients, in diabetics, and in certain racial and ethnic groups, it may be prudent to treat patients in high-risk groups during the primary infection, before dissemination takes place. In the past some authorities recommended a treatment course to a total dose of 500 to $2000 \mathrm{mg}$ of amphotericin B [80]. Similarly, many experts believed that all patients with pulmonary disease that is severe or persists beyond a few weeks should receive amphotericin B to approximately the same total dose to prevent local pulmonary 
progression and to prevent dissemination. In current practice, many such patients (and also less symptomatic patients with pulmonary coccidioidomycosis of shorter duration) are often given fluconazole for 3-6 months, reserving AMB for patients with diffuse infiltrates and women in the third trimester of pregnancy. These recommendations are based on expert opinion and observational studies.

Amphotericin B is likely the best treatment for persistent pulmonary coccidioidomycosis. Because of their lesser toxicity, oral azoles are often tried. About two thirds of patients have clinical improvement with azole therapy, but many relapse when the course of treatment is finished. Ketoconazole was used first. Currently fluconazole and itraconazole are being used. Voriconazole will likely be evaluated in the future.

Disseminated coccidioidomycosis requires prompt and aggressive treatment. Unfortunately, amphotericin B is not as effective for disseminated coccidioidomycosis as it is for disseminated histoplasmosis or blastomycosis. The standard dose of amphotericin B is 2500 to $3000 \mathrm{mg}$ given over many weeks or months. If necessary, much larger total doses may be given [81]. Daily doses of amphotericin B (usually 40 to $50 \mathrm{mg}$ ) are given while the patient is acutely ill. When the patient stabilizes, frequency should be reduced to three times weekly. Currently disseminated disease without CNS involvement should be treated with fluconazole or itraconazole first, especially in mild to moderate cases. AMB should be reserved for severe disease or treatment failure.

Fluconazole and itraconazole are now azoles of choice for nonmeningeal disseminated coccidioidomycosis. Neither is perfect for difficult cases for which even amphotericin B is often only suppressive. Long-term therapy is often required, extending to years or even indefinitely. Fluconazole has the advantage of better absorption, less gastrointestinal upset, and better penetration of the central nervous system. In a recently published randomized controlled trial, oral fluconazole and itraconazole were compared for treatment of non-meningeal coccidioidomycosis. Soft tissue dissemination responded best. Overall, itraconazole was somewhat more effective than fluconazole, producing response in $63 \%$ of the patients vs. $50 \%$ response in fluconazole treated patients $(\mathrm{p}=0.08)$. Among patients with skeletal infections, itraconazole was clearly superior, $(\mathrm{p}=0.05)$ [82]. Some difficult cases of bone, lymph node, and soft-tissue coccidioidomycosis may be best managed with surgical drainage of focal 
abscesses, a 1000 to $2000 \mathrm{mg}$ course of amphotericin B, and a prolonged course of itraconazole or fluconazole.

As might be expected, the treatment of disseminated coccidioidomycosis in AIDS is particularly difficult. Because of the rapid tempo of the disease, amphotericin B should be used initially, especially if the patient is severely ill. If the clinical course stabilizes, it is reasonable to switch to fluconazole for long-term suppression. Prognosis is poor. Even with prompt diagnosis and treatment, up to $40 \%$ of severely immunosuppressed patients die during the initial hospitalization. Other patients, usually with lesser degrees of immunosuppression, respond well to treatment[70], [71] .

Meningeal coccidioidomycosis is a major therapeutic challenge. The standard therapy in the past included a course of 2000 to $3000 \mathrm{mg}$ systemic amphotericin therapy plus intensive and lengthy intrathecal (by lumbar or cisternal route) AMB therapy [63]. Intrathecal (or, less commonly, intraventricular via surgically placed reservoir [83]) AMB in doses between 0.25 and $1 \mathrm{mg}$ was injected two to three times weekly until symptoms and cerebrospinal fluid pleocytosis resolve. Even after the patient had apparently recovered fully and cerebrospinal fluid pleocytosis had resolved, most authorities recommended continued injections of amphotericin to prevent relapse, first weekly and then at longer intervals. Relapses were common, but, with careful management, lengthy remissions could be obtained.

Because of the toxicity of this once standard approach to coccidioidomycotic meningitis, fluconazole has been evaluated as primary therapy for stable patients and as suppressive therapy after initial response to amphotericin B for more severely ill patients. Most patients respond favorably to fluconazole and maintain good clinical function. Dosage is 400 to $600 \mathrm{mg} /$ daily or even higher. Therapy has to be continued long term, likely indefinitely [84]. Recently anecdotal reports have shown favorable response to voriconazole and this agent will undoubtedly be tried in various forms of coccidioidomycosis, including meningitis. A drug with potency and wide spectrum of itraconazole but with tissue penetration like fluconazole seems especially attractive for an treatment resistant illness with high incidence of meningeal spread. However clinical data is sparse.

Severely ill patients with both nonmeningeal and meningeal disease were previously treated with intravenous and intrathecal amphotericin B. Now 
they are sometimes treated with intravenous amphotericin B for faster, more effective initial therapy of the nonmeningeal disease and with fluconazole to control the central nervous system infection. Amphotericin B is continued to clinical improvement and fluconazole indefinitely.

Newer antifungal agents are being developed; their potential role in coccidioidomycosis is uncertain. As mentioned voriconazole has some promise because it has better CNS penetration than itraconazole - and yet may retain the potency advantage of itraconazole over fluconazole which has been demonstrated in non-meningeal disseminated disease.

\section{REFERENCES}

1. Goodwin, R.A., Jr. and R.M. Des Prez, State of the art: histoplasmosis. Am Rev Respir Dis, 1978. 117(5): p. 929-56.

2. Sarosi, G.A. and S.F. Davies, Blastomycosis. Am Rev Respir Dis, 1979. 120(4): p. 911-38.

3. Drutz, D.J. and A. Catanzaro, Coccidioidomycosis. Part I. Am Rev Respir Dis, 1978. 117(3): p. 559-85.

4. Davies, S.F., M. Khan, and G.A. Sarosi, Disseminated histoplasmosis in immunologically suppressed patients. Occurrence in a nonendemic area. Am J Med, 1978. 64(1): p. 94-100.

5. Goodwin, R.A., Jr., et al., Disseminated histoplasmosis: clinical and pathologic correlations. Medicine (Baltimore), 1980. 59(1): p. 1-33.

6. Manfredi, R., et al., Histoplasmosis capsulati and duboisii in Europe: the impact of the HIV pandemic, travel and immigration. Eur J Epidemiol, 1994. 10(6): p. 675-81.

7. Edwards, L.B., et al., An atlas of sensitivity to tuberculin, PPD-B, and histoplasmin in the United States. Am Rev Respir Dis. Vol. 99. 1969. Suppl:1132.

8. Hammerman, K.J., K.E. Powell, and F.E. Tosh, The incidence of hospitalized cases of systemic mycotic infections. Sabouraudia, 1974. 12(1): p. 33-45.

9. Wheat, L.J., et al., A large urban outbreak of histoplasmosis: clinical features. Ann Intern Med, 1981. 94(3): p. 331-7.

10. Wheat, L.J., et al., Disseminated histoplasmosis in the acquired immune deficiency syndrome: clinical findings, diagnosis and treatment, and review of the literature. Medicine (Baltimore), 1990. 69(6): p. 361-74. 
11. Procknow, J.J., M.I. Page, and C.G. Loosli, Early pathogenesis of experimental histoplasmosis. Arch Pathol, 1960. 69: p: 413-26.

12. Zhou, P., G. Miller, and R.A. Seder, Factors involved in regulating primary and secondary immunity to infection with Histoplasma capsulatum: TNF-alpha plays a critical role in maintaining secondary immunity in the absence of IFNgamma. J Immunol, 1998. 160(3): p. 1359-68.

13. Allendoerfer, R., G.P. Biovin, and G.S. Deepe, Jr., Modulation of immune responses in murine pulmonary histoplasmosis. J Infect Dis, 1997. 175(4): p. 905-14.

14. Allendorfer, R., G.D. Brunner, and G.S. Deepe, Jr., Complex requirements for nascent and memory immunity in pulmonary histoplasmosis. J Immunol, 1999. 162(12): p. 7389-96.

15. Allendoerfer, R. and G.S. Deepe, Jr., Intrapulmonary response to Histoplasma capsulatum in gamma interferon knockout mice. Infect Immun, 1997. 65(7): p. 2564-9.

16. Wood, K.L., et al., Histoplasmosis after treatment with anti-tumor necrosis factor-alpha therapy. Am J Respir Crit Care Med, 2003. 167(9): p. 1279-82.

17. Straub, M. and J. Schwarz, The healed primary complex in histoplasmosis. Am J Clin Pathol, 1955. 25(7): p. 727-41.

18. Wheat, J., Histoplasmosis. Experience during outbreaks in Indianapolis and review of the literature. Medicine (Baltimore), 1997. 76(5): p. 339-54.

19. Conces, D.J., Jr., et al., Disseminated histoplasmosis in AIDS: findings on chest radiographs. AJR Am J Roentgenol, 1993. 160(1): p. 15-9.

20. Davies, S.F., R.W. McKenna, and G.A. Sarosi, Trephine biopsy of the bone marrow in disseminated histoplasmosis. Am J Med, 1979. 67(4): p. 617-22.

21. Wheat, L.J., T.G. Slama, and M.L. Zeckel, Histoplasmosis in the acquired immune deficiency syndrome. Am J Med, 1985. 78(2): p. 203-10.

22. Johnson, P.C., R.J. Hamill, and G.A. Sarosi, Clinical review: progressive disseminated histoplasmosis in the AIDS patient. Semin Respir Infect, 1989. 4(2): p. 139-46.

23. Wheat, L.J., et al., Risk factors for disseminated or fatal histoplasmosis. Analysis of a large urban outbreak. Ann Intern Med, 1982. 96(2): p. 159-63.

24. Sarosi, G.A., et al., Disseminated histoplasmosis: results of long-term followup. A center for disease control cooperative mycoses study. Ann Intern Med, 1971. 75(4): p. 511-6.

25. Wheat, L.J., B.E. Batteiger, and B. Sathapatayavongs, Histoplasma capsulatum infections of the central nervous system. A clinical review. Medicine (Baltimore), 1990. 69(4): p. 244-60. 
26. Hawkins, S.S., D.W. Gregory, and R.H. Alford, Progressive disseminated histoplasmosis; favorable response to ketoconazole. Ann Intern Med, 1981. 95(4): p. 446-9.

27. Vail, G.M., et al., Incidence of histoplasmosis following allogeneic bone marrow transplant or solid organ transplant in a hyperendemic area. Transpl Infect Dis, 2002. 4(3): p. 148-51.

28. Davies, S.F., Serodiagnosis of histoplasmosis. Semin Respir Infect, 1986. 1(1): p. 9-15.

29. Prechter, G.C. and U.B. Prakash, Bronchoscopy in the diagnosis of pulmonary histoplasmosis. Chest, 1989. 95(5): p. 1033-6.

30. Wheat, L.J., et al., Diagnosis of histoplasmosis in patients with the acquired immunodeficiency syndrome by detection of Histoplasma capsulatum polysaccharide antigen in bronchoalveolar lavage fluid. Am Rev Respir Dis, 1992. 145(6): p. 1421-4.

31. Salzman, S.H., R.L. Smith, and C.P. Aranda, Histoplasmosis in patients at risk for the acquired immunodeficiency syndrome in a nonendemic setting. Chest, 1988. 93(5): p. 916-21.

32. Wheat, J., et al., Cross-reactivity in Histoplasma capsulatum variety capsulatum antigen assays of urine samples from patients with endemic mycoses. Clin Infect Dis, 1997. 24(6): p. 1169-71.

33. Wheat, L.J., et al., Histoplasmosis relapse in patients with AIDS: detection using Histoplasma capsulatum variety capsulatum antigen levels. Ann Intern Med, 1991. 115(12): p. 936-41.

34. Wheat, L.J., et al., Effect of successful treatment with amphotericin B on Histoplasma capsulatum variety capsulatum polysaccharide antigen levels in patients with AIDS and histoplasmosis. Am J Med, 1992. 92(2): p. 153-60.

35. Dismukes, W.E., et al., Itraconazole therapy for blastomycosis and histoplasmosis. NIAID Mycoses Study Group. Am J Med, 1992. 93(5): p. 48997.

36. Wheat, J., et al., Prevention of relapse of histoplasmosis with itraconazole in patients with the acquired immunodeficiency syndrome. The National Institute of Allergy and Infectious Diseases Clinical Trials and Mycoses Study Group Collaborators. Ann Intern Med, 1993. 118(8): p. 610-6.

37. Hecht, P.M., et al., Itraconazole maintenance treatment for histoplasmosis in AIDS: a prospective, multicenter trial. J Acquir Immune Defic Syndr Hum Retrovirol, 1997. 16(2): p. 100-7.

38. Goldman, M., et al., Safety of Discontinuation of Maintenance Therapy for Disseminated Histoplasmosis after Immunologic Response to Antiretroviral Therapy: AIDS Clinical Trials Group Study A5038. Clinical Iinfectious Diseases, 2004:38 (15 May). In press . 
39. Wheat, J., et al., Itraconazole treatment of disseminated histoplasmosis in patients with the acquired immunodeficiency syndrome. AIDS Clinical Trial Group. Am J Med, 1995. 98(4): p. 336-42.

40. Wheat, J., et al., Treatment of histoplasmosis with fluconazole in patients with acquired immunodeficiency syndrome. National Institute of Allergy and Infectious Diseases Acquired Immunodeficiency Syndrome Clinical Trials Group and Mycoses Study Group. Am J Med, 1997. 103(3): p. 223-32.

41. Johnson, P.C., et al., Safety and efficacy of liposomal amphotericin B compared with conventional amphotericin B for induction therapy of histoplasmosis in patients with AIDS. Ann Intern Med, 2002. 137(2): p. 105-9.

42. Furcolow, M.L., et al., Prevalence and incidence studies of human and canine blastomycosis. 1. Cases in the United States, 1885-1968. Am Rev Respir Dis, 1970. 102(1): p. 60-7.

43. Kepron, M.W., et al., North American blastomycosis in Central Canada. A review of 36 cases. Can Med Assoc J, 1972. 106(3): p. 243-6.

44. Tosh, F.E., et al., A common source epidemic of North American blastomycosis. Am Rev Respir Dis, 1974. 109(5): p. 525-9.

45. Greenberg, S.B., Serious waterborne and wilderness infections. Crit Care Clin, 1999. 15(2): p. 387-414.

46. Baumgardner, D. J. and K. Brockman, Epidemiology of human blastomycosis in Vilas County, Wisconsin. II: 1991-1996. Wmj, 1998. 97(5): p. 44-7.

47. Sarosi, G.A., et al., Canine blastomycosis as a harbinger of human disease. Ann Intern Med, 1979. 91(5): p. 733-5.

48. Witorsch, P. and J.P. Utz, North American blastomycosis: a study of 40 patients. Medicine (Baltimore), 1968. 47(3): p. 169-200.

49. Klein, B.S., et al., Isolation of Blastomyces dermatitidis in soil associated with a large outbreak of blastomycosis in Wisconsin. N Engl J Med, 1986. 314(9): p. 529-34.

50. Klein, B.S., J.M. Vergeront, and J.P. Davis, Epidemiologic aspects of blastomycosis, the enigmatic systemic mycosis. Semin Respir Infect, 1986. 1(1): p. 29-39.

51. Abernathy, R.S., Clinical manifestations of pulmonary blastomycosis. Ann Intern Med, 1959. 51: p. 707-27.

52. Laskey, W. and G.A. Sarosi, The radiological appearance of pulmonary blastomycosis. Radiology, 1978. 126(2): p. 351-7.

53. Davies, S. and G. Sarosi, Clinical manifestations and management of blastomycosis in the compromised patient. In Warnock DW, Richard MD (eds): Fungal Infection in the Compromised Patient. New York: John Wiley \& Sons, 1982, 1982: p. 215-229. 
54. Pappas, P.G., et al., Blastomycosis in patients with the acquired immunodeficiency syndrome. Ann Intern Med, 1992. 116(10): p. 847-53.

55. Trumbull, M.L. and T.M. Chesney, The cytological diagnosis of pulmonary blastomycosis. Jama, 1981. 245(8): p. 836-8.

56. Martynowicz, M.A. and U.B. Prakash, Pulmonary blastomycosis: an appraisal of diagnostic techniques. Chest, 2002. 121(3): p. 768-73.

57. Lemos, L.B., M. Guo, and M. Baliga, Blastomycosis: organ involvement and etiologic diagnosis. A review of 123 patients from Mississippi. Ann Diagn Pathol, 2000. 4(6): p. 391-406.

58. Gonyea, E.F., The spectrum of primary blastomycotic meningitis: a review of central nervous system blastomycosis. Ann Neurol, 1978. 3(1): p. 26-39.

59. Kravitz, G.R., et al., Chronic blastomycotic meningitis. Am J Med, 1981. 71(3): p. 501-5.

60. Flynn, N.M., et al., An unusual outbreak of windborne coccidioidomycosis. N Engl J Med, 1979. 301(7): p. 358-61.

61. Werner, S.B., et al., An epidemic of coccidioidomycosis among archeology students in northern California. N Engl J Med, 1972. 286(10): p. 507-12.

62. Drutz, D.J. and A. Catanzaro, Coccidioidomycosis. Part II. Am Rev Respir Dis, 1978. 117(4): p. 727-71.

63. Bouza, E., et al., Coccidioidal meningitis. An analysis of thirty-one cases and review of the literature. Medicine (Baltimore), 1981. 60(3): p. 139-72.

64. Bayer, A.S., et al., Unusual syndromes of coccidioidomycosis: diagnostic and therapeutic considerations; a report of 10 cases and review of the English literature. Medicine (Baltimore), 1976. 55(2): p. 131-52.

65. Bayer, A.S., Fungal pneumonias; pulmonary coccidioidal syndromes (Part I). Primary and progressive primary coccidioidal pneumonias -- diagnostic, therapeutic, and prognostic considerations. Chest, 1981. 79(5): p. 575-83.

66. Winn, W.A., A long term study of 300 patients with cavitary-abscess lesions of the lung of coccidioidal origin. An analytical study with special reference to treatment. Dis Chest, 1968. 54: p. Suppl 1:268+.

67. Rutala, P.J. and J.W. Smith, Coccidioidomycosis in potentially compromised hosts: the effect of immunosuppressive therapy in dissemination. Am J Med Sci, 1978. 275(3): p. 283-95.

68. Cohen, I.M., et al., Coccidioidomycosis in renal replacement therapy. Arch Intern Med, 1982. 142(3): p. 489-94.

69. Blair, J.E., D.D. Douglas, and D.C. Mulligan, Early results of targeted prophylaxis for coccidioidomycosis in patients undergoing orthotopic liver transplantation within an endemic area. Transpl Infect Dis, 2003. 5(1): p. 3-8. 
70. Bronnimann, D.A., et al., Coccidioidomycosis in the acquired immunodeficiency syndrome. Ann Intern Med, 1987. 106(3): p. 372-9.

71. Fish, D.G., et al., Coccidioidomycosis during human immunodeficiency virus infection. A review of 77 patients. Medicine (Baltimore), 1990. 69(6): p. 38491.

72. Warlick, M.A., S.F. Quan, and R.E. Sobonya, Rapid diagnosis of pulmonary coccidioidomycosis. Cytologic v potassium hydroxide preparations. Arch Intern Med, 1983. 143(4): p. 723-5.

73. Wallace, J.M., et al., Flexible fiberoptic bronchoscopy for diagnosing pulmonary coccidioidomycosis. Am Rev Respir Dis, 1981. 123(3): p. 286-90.

74. Polesky, A., et al., Airway coccidioidomycosis--report of cases and review. Clin Infect Dis, 1999. 28(6): p. 1273-80.

75. Mahaffey, K.W., et al., Unrecognized coccidioidomycosis complicating Pneumocystis carinii pneumonia in patients infected with the human immunodeficiency virus and treated with corticosteroids. A report of two cases. Arch Intern Med, 1993. 153(12): p. 1496-8.

76. Sobonya, R.E., et al., Detection of fungi and other pathogens in immunocompromised patients by bronchoalveolar lavage in an area endemic for coccidioidomycosis. Chest, 1990. 97(6): p. 1349-55.

77. Sarosi, G.A., et al., Rapid diagnostic evaluation of bronchial washings in patients with suspected coccidioidomycosis. Semin Respir Infect, 2001. 16(4): p. 238-41.

78. Standard, P.G. and L. Kaufman, Immunological procedure for the rapid and specific identification of Coccidioides immitis cultures. J Clin Microbiol, 1977. 5(2): p. 149-53.

79. Smith, C.E., M.T. Saito, and S.A. Simons, Pattern of 39,500 serologic tests in coccidioidomycosis. J Am Med Assoc, 1956. 160(7): p. 546-52.

80. Galgiani, J.N., et al., Practice guideline for the treatment of coccidioidomycosis. Infectious Diseases Society of America. Clin Infect Dis, 2000. 30(4): p. 658-61.

81. Bennett, J.E., Chemotherapy of systemic mycoses (first of two parts). N Engl J Med, 1974. 290(1): p. 30-2.

82. Galgiani, J.N., et al., Comparison of oral fluconazole and itraconazole for progressive, nonmeningeal coccidioidomycosis. A randomized, double-blind trial. Mycoses Study Group. Ann Intern Med, 2000. 133(9): p. 676-86.

83. Diamond, R.D. and J.E. Bennett, A subcutaneous reservoir for intrathecal therapy of fungal meningitis. N Engl J Med, 1973. 288(4): p. 186-8.

84. Dewsnup, D.H., et al., Is it ever safe to stop azole therapy for Coccidioides immitis meningitis? Ann Intern Med, 1996. 124(3): p. 305-10. 